\title{
Feasibility of a Web-Based Implementation Intervention to Improve Child Dietary Intake in Early Childhood Education and Care: Pilot Randomized Controlled Trial
}

Courtney Barnes ${ }^{1,2,3,4}$, PhD; Sze Lin Yoong ${ }^{1,2,3,4,5}$, PhD; Nicole Nathan ${ }^{1,2,3,4}$, PhD; Luke Wolfenden ${ }^{1,2,3,4}$, PhD; Taya

Wedesweiler $^{1}$, BHlth PhysEd (Hons); Jayde Kerr ${ }^{1}$, BNutrDiet; Dianne S Ward ${ }^{6,7}$, EdD; Alice Grady ${ }^{1,2,3,4}$, PhD

${ }^{1}$ Hunter New England Population Health, Newcastle, Australia

${ }^{2}$ School of Medicine and Public Health, University of Newcastle, Callaghan, Australia

${ }^{3}$ Hunter Medical Research Institute, New Lambton, Australia

${ }^{4}$ Priority Research Centre for Health Behaviour, University of Newcastle, Callaghan, Australia

${ }^{5}$ School of Health Sciences, Swinburne University of Technology, Melbourne, Australia

${ }^{6}$ Department of Nutrition, Gillings School of Global Public Health, University of North Carolina, Chapel Hill, NC, United States

${ }^{7}$ Center for Health Promotion and Disease Prevention, University of North Carolina, Chapel Hill, NC, United States

\section{Corresponding Author:}

Courtney Barnes, PhD

Hunter New England Population Health

Locked Bag 10, Wallsend

Newcastle, 2287

Australia

Phone: 610249246678

Email: courtney.barnes@health.nsw.gov.au

\begin{abstract}
Background: Internationally, the implementation of evidence-based healthy eating policies and practices within early childhood education and care (ECEC) settings that encourage children's healthy diet is recommended. Despite the existence of evidence-based healthy eating practices, research indicates that current implementation rates are inadequate. Web-based approaches provide a potentially effective and less costly approach to support ECEC staff with implementing nutrition policies and practices.

Objective: The broad aim of this pilot randomized controlled trial is to assess the feasibility of assessing the impact of a web-based program together with health promotion officer (HPO) support on ECEC center implementation of healthy eating policies and practices. Specifically, we seek to describe the completion rate of study evaluation processes (participant consent and data collection rates); examine ECEC center uptake, acceptability, and appropriateness of the intervention and implementation strategies; understand the potential cost of delivering and receiving implementation support strategies; and describe the potential impact of the web-based intervention on the implementation of targeted healthy eating practices among centers in the intervention group.

Methods: A 6-month pilot implementation trial using a cluster-randomized controlled trial design was conducted in 22 ECEC centers within the Hunter New England region of New South Wales, Australia. Potentially eligible centers were distributed a recruitment package and telephoned by the research team to assess eligibility and obtain consent. Centers randomly allocated to the intervention group received access to a web-based program, together with HPO support (eg, educational outreach visit and local technical assistance) to implement 5 healthy eating practices. The web-based program incorporated audit with feedback, development of formal implementation blueprints, and educational materials to facilitate improvement in implementation. The centers allocated to the control group received the usual care.
\end{abstract}

Results: Of the 57 centers approached for the study, 22 (47\%) provided consent to participate. Data collection components were completed by $100 \%$ (22/22) of the centers. High uptake for implementation strategies provided by HPOs (10/11, 91\% to $11 / 11$, $100 \%)$ and the web-based program $(11 / 11,100 \%)$ was observed. At follow-up, intervention centers had logged on to the program at an average of 5.18 (SD 2.52) times. The web-based program and implementation support strategies were highly acceptable $(10 / 11,91 \%$ to $11 / 11,100 \%)$. Implementation of 4 healthy eating practices improved in the intervention group, ranging from $19 \%(2 / 11)$ to $64 \%(7 / 11)$. 
Conclusions: This study provides promising pilot data to warrant the conduct of a fully powered implementation trial to assess the impact of the program on ECEC healthy eating practice implementation.

Trial Registration: Australian New Zealand Clinical Trials Registry (ANZCTR) ACTRN12619001158156; https://www.anzctr.org.au/Trial/Registration/TrialReview.aspx?id=378099

International Registered Report Identifier (IRRID)： RR2-10.1186/s40814-020-00707-w

(J Med Internet Res 2021;23(12):e25902) doi: 10.2196/25902

\section{KEYWORDS}

childcare center; web-based; nutrition; healthy eating; randomized controlled trial; intervention; implementation

\section{Introduction}

\section{Background}

Poor dietary intake in early childhood, including inadequate intake of fruit and vegetables and excessive intake of discretionary foods (high in added sugar, sodium, and saturated fat), is a leading contributor to the development of childhood overweight, obesity, cardiovascular disease, and specific types of cancers $[1,2]$. Globally, preschool aged children do not meet national dietary recommendations for intake of fruit and vegetable servings, while overconsuming discretionary food items [2-5]. As dietary behaviors developed during childhood are known to track into adulthood [6], population-level interventions (ie, interventions targeting a large proportion of the population) to improve child nutrition are recommended [7,8]. Early childhood education and care (ECEC) is a promising setting for interventions aimed at improving children's nutrition behaviors, as they provide access to a large proportion of children [3,9] for prolonged periods [10] during a crucial period of development [11].

Systematic review evidence has identified numerous ECEC-based interventions effective in improving child nutrition behaviors [12] and center nutrition environments [13], including the implementation of evidence-based ECEC practices associated with improved child dietary intake in care $[13,14]$. The implementation of such evidence-based practices is recommended within national and international ECEC guidelines and includes the provision of healthy foods, positive educator feeding practices (eg, role modeling healthy food choices), and developing center nutrition policies, which detail center strategies and guidelines to enforce the implementation of healthy eating practices [15-17]. However, despite the existence of such guidelines, numerous studies have indicated that the current implementation of evidence-based healthy eating practices is inadequate [18-21].

A recent Cochrane systematic review identified that multicomponent implementation strategies, including researcher delivered face-to-face nutrition education sessions and ongoing support, can produce small but significant improvements in the implementation of healthy eating practices in ECEC centers [13]. Although potentially effective, there are significant challenges in delivering such interventions at scale (ie, to a large number of ECEC centers), including financial and resource burden on centers and the lack of alignment with center capabilities and infrastructure [13]. Web-based modalities provide a potentially effective and less costly approach to implementing nutrition interventions at scale in this setting. Previous research suggests that the use of such modalities to deliver support to center staff is highly acceptable and fits within the existing center infrastructure (eg, access to computers and internet) [12,22,23]. In addition, these modalities can reach a large proportion of the population [24] and have been associated with improvement in a range of provider behaviors and implementation outcomes in previous research delivered outside the ECEC setting [25,26].

Recent trials examining the impact of web-based interventions on ECEC healthy eating practices have been conducted within menu-based centers (ie, centers that provide food to children). A randomized controlled trial (RCT) conducted in 54 Australian childcare centers evaluated the impact of a web-based menu planning program on center compliance with sector dietary guidelines [27]. Results of the RCT found statistically significant improvements in the servings of core food groups and child diet intake; however, the intervention had nonsignificant improvements in the primary outcome of menu compliance with all food groups. The study reported variable levels of engagement with the web-based program, despite the high uptake of implementation support strategies and high acceptability of the intervention and implementation support provided [27]. In addition, the web-based intervention was deemed a cost-effective alternative to traditional menu planning approaches [23]. Within the United States, a pilot RCT conducted in 31 centers evaluated the impact of the web-based Nutrition and Physical Activity Self-Assessment for Child Care (Go-Nutrition and Physical Activity Self-Assessment for Child Care [Go-NAPSACC]) program on center nutrition environments [28]. Despite improvements in food and beverages provided within intervention centers, no statistically significant differences in center nutrition environments were reported at follow-up [28]. Center engagement with the web-based program was not reported; however, the uptake of the implementation support strategies was high among intervention centers. Findings from the process evaluation indicated that a lack of computer literacy among center staff and the need for additional technical support were barriers to program use [28]. Despite these studies showing promise, no RCTs examining the impact of web-based interventions on ECEC healthy eating practices within lunchbox centers (ie, where parents pack foods for children to consume in care) have been conducted.

\section{Objectives}

Given the differences between menu-based and lunchbox centers, there is a need to understand whether such interventions 
are feasible in the ECEC setting. Feasibility studies are recommended as they allow researchers to collect data to determine whether an intervention is appropriate for more robust testing and to pilot-test recruitment and data collection methods and tools to inform a larger trial [29]. Thus, the aim of this pilot RCT is to determine the feasibility of conducting a fully powered implementation trial assessing the impact of a web-based program together with health promotion officer (HPO) support, on childcare center implementation of healthy eating policies and practices. Specifically, we seek to (1) describe the completion of study evaluation processes (participant consent and data collection rates); (2) examine ECEC center uptake, acceptability, and appropriateness of the intervention and implementation strategies; (3) understand the potential cost of delivering and receiving the implementation strategies; and (4) describe the potential impact of the web-based intervention on the implementation of healthy eating practices among centers in the intervention group.

\section{Methods}

\section{Registration and Ethics Approval}

This trial was prospectively registered with the Australian New Zealand Clinical Trials Registry (ACTRN12619001158156) and followed the CONSORT reporting guidelines for pilot and feasibility studies [30]. Ethical approval for the trial was obtained from Hunter New England (HNE; HNE approval 06/07/26/4.04) and the University of Newcastle (approval H-2008-0343) Human Research Ethics Committees.

This trial was originally designed as a cluster RCT using an effectiveness-implementation hybrid type-II design. A hybrid effectiveness-implementation design was used to pilot the potential impact and assess the feasibility of an implementation intervention, while assessing the effectiveness of the intervention in improving child dietary intake in care as described by Curran et al [31]. Owing to COVID-19 precluding center site visits to conduct follow-up data collection, we were unable to undertake child lunchbox and dietary assessments and, as such, have not been reported. Therefore, this paper reports on the pilot implementation outcomes that could still be evaluated at follow-up and were specified in the trial registration and protocol.

\section{Study Design and Setting}

A protocol detailing the study design and methodology has been published elsewhere [32]. In brief, a pilot implementation trial using a cluster RCT design was conducted in center-based childcare centers within the HNE region of New South Wales, Australia. The HNE region is socioeconomically and geographically diverse, encompassing metropolitan, regional, and remote communities, with a population of over 920,000 residents [33]. Approximately 422 center-based childcare centers, including preschools and long day care, are located within the HNE region, which typically enroll children aged 0-6 years for an average of 21 hours per week [10,34].

\section{Participant Eligibility and Recruitment}

\section{Centers}

Centers were eligible to participate in the trial if (1) they enrolled $>20$ children per day, (2) had internet access, (3) parents provided food for children to consume while attending care (ie, centers did not provide food), (4) they did not participate in any other healthy eating or physical activity intervention, and (5) they were not fully compliant with healthy eating practices (ie, not implementing all 5 practices) specified in the NSW state obesity-prevention program (ie, Munch \& Move) targeted by the intervention, according to the NSW Ministry of Health data monitoring [35]. Centers were ineligible if they were a mobile preschool or family day care center, did not cater to children aged 2-5 years, catered exclusively for children requiring specialist care, or were classified as an NSW Department of Education center owing to differing operational characteristics.

A list of potentially eligible centers located within the HNE region was obtained from the NSW Ministry of Health [35]. One member of the research team with experience recruiting centers to health promotion trials led the recruitment process and monitored consent rates. First, centers were progressively distributed a recruitment package consisting of a study information statement and consent form in random order. Second, the research team member leading recruitment telephoned centers to discuss study details, assess eligibility, and request consent for study participation $[19,36]$. The centers continued to be contacted until the required number $(n=22)$ consented. During the telephone call, the research team member also scheduled a 2-day baseline data collection site visit for consenting centers. Recruitment for the study was conducted between August 2019 and October 2019.

\section{Children}

For children to be eligible to participate, they were required to (1) have written consent from a parent or guardian, (2) be between the ages of 2 and 5 years, (3) be enrolled to attend the center on at least one of the scheduled days of data collection, and (4) not have a dietary restriction requiring specialized tailoring of their diet (eg, allergies or intellectual or physical disability).

Approximately 2 weeks before the baseline data collection site visit, centers were asked to distribute consent forms and information statements to parents via usual communication methods, including email, communication apps, and child pigeonholes. Trained research assistants with experience in recruitment and data collection attended the childcare centers approximately one week before the site visit and on the days of the site visits to request written consent from parents for their children to participate in the study.

\section{Randomization and Blinding}

Following baseline data collection, centers were randomly allocated to the intervention or control group, stratified by center socioeconomic status (SES). On the basis of center postcodes, the 2016 Socio-Economic Indexes for Areas was used to classify centers as being located in the least disadvantaged (high SES) or most disadvantaged (low SES) areas [37]. Center postcodes 
ranked in the top $50 \%$ of NSW were classified as least disadvantaged and the lower $50 \%$ of postcodes as the most disadvantaged. The centers were also stratified by those with a high number of Aboriginal child enrollments (defined as those with $>10 \%$ Aboriginal child enrollments), in a 1:1 ratio through a block randomization procedure (block sizes 2 or 4 ) conducted by an independent blinded statistician. Given the nature of the intervention (ie, intervention centers were provided access to a web-based program), the centers were not blinded to group allocation. Data collectors were not blinded to group allocation at follow-up.

\section{Intervention}

The intervention aimed to improve the implementation of childcare center-level healthy eating practices. The practices targeted within the intervention are recommended by the NSW state obesity-prevention program (ie, Munch \& Move) [17] as well as national and international guidelines, acknowledging the association between such practices and improved child dietary intake in care $[15,16]$. Specifically, the practices included the following:

1. Supporting families to provide healthier foods consistent with dietary guidelines: center staff within the intervention group were provided with healthy eating information and resources via the web-based program and were asked to disseminate these to families via usual center communication methods, such as mobile apps, email, and written information, twice during the intervention period. Center staff were also asked to monitor children's lunchboxes daily for consistency with sector-specific dietary guidelines and provide feedback to parents.

2. Provision of intentional healthy eating learning experiences (eg, gardening and cooking lessons): center staff were asked to provide children with intentional healthy eating learning experiences at least twice per week.

3. Using feeding practices that support children's healthy eating (eg, educator role modeling healthy food choices): center staff were asked to provide encouragement to children to promote healthy eating and trying new foods at every meal and snack occasion. Center staff were also asked to role model consuming healthy food choices and avoid the use of foods to encourage desired behavior.

4. Staff participating in professional development targeting healthy eating: center staff were asked to have at least 50\% of the staff to participate in web-based training opportunities specific to staff healthy eating behaviors and center practices.

5. Having a comprehensive written nutrition policy that outlines key healthy eating practices: centers were asked to develop or modify existing nutrition policies to document procedures and strategies to facilitate the implementation of healthy eating practices to improve child diet.

A detailed description of these practices is provided in the study protocol [32].
A web-based program, known as Childcare Electronic Assessment Tool and Support (EATS), was developed by the research team to support center implementation of the 5 targeted healthy eating practices. The centers allocated to the intervention group were provided with free access to the web-based program. The intervention was developed by behavioral science researchers, HPOs, state government representatives, and end users from the ECEC setting, including nominated supervisors and educators.

The Behavior Change Wheel (BCW) [38] was used to guide the development and selection of implementation strategies to support center staff in achieving behavior change. During this process, barriers and enablers to center behavior change identified through a literature review and engagement with ECEC staff and stakeholders were mapped to specific behavior change techniques (BCTs) within the BCW [38]. A suite of implementation strategies, defined according to the expert recommendations for implementing change taxonomy, were then selected to action the BCTs within the intervention [39]. The content and implementation strategies within Childcare EATS were selected to ensure user (ie, center staff) engagement, including self-assessment and action planning components to allow center nominated supervisors to reflect on current practice and housed educational resources to facilitate improvements in staff behavior and center processes. The features of the program were developed to integrate within existing center procedures, (eg, the ability to download feedback from the self-assessment quiz) and national assessment and rating standards (eg, the development of action plans as evidence within quality improvement plans). Extensive pilot testing was undertaken with ECEC staff through face-to-face meetings with HPOs to ensure that the functionality and content of Childcare EATS was appropriate and that any potential barriers to program use were addressed. Limitations from previous web-based interventions conducted within the ECEC setting, including low staff computer literacy, need for ongoing technical support, and competing priorities of ECEC staff were also considered during the development of the program $[28,40]$.

Implementation strategies additional to those embedded within the web-based program identified via the $\mathrm{BCW}$ process above were used by HPOs who work within the state local health districts to deliver health promotion initiatives within community-based settings such as childcare centers. The HPOs received a training session and implementation manual before delivering the intervention. In addition, HPOs conducted 2 pilot training sessions, with both internal (health service staff with extensive experience supporting ECEC centers to implement obesity prevention initiatives) and external (ECEC center staff) stakeholders. The application of these implementation strategies within the intervention is summarized in Table 1 using the Proctor framework [41] to enable replication. 
Table 1. Implementation strategies and behavior change techniques used within the web-based intervention.

\begin{tabular}{lll}
\hline $\begin{array}{l}\text { Mode of delivery and implementation } \\
\text { strategy according to ERIC }{ }^{\mathrm{a}}[39]\end{array}$ & Application of the implementation strategy according to Proctor [41] & $\begin{array}{l}\text { Behavior change technique ac- } \\
\text { tioned via the implementation } \\
\text { strategy }\end{array}$ \\
\hline
\end{tabular}

\section{Web-based program}

Audit with feedback

Develop a formal implementation blueprint

Distribute educational materials

- Actor: web-based program

- Action: the Childcare EATS program housed a suite of materials to assist center implementation of the targeted practices, including factsheets and resources to facilitate communication with parents; educational materials to improve staff knowledge; example healthy eating learning experiences; professional development and policy templates.

- Target: nominated supervisors and center champions to increase staff member knowledge and abilities to implement practices

- Temporality: commencement of the intervention. Centers were encouraged to access resources immediately following action planning (development of a formal implementation blueprint).

- Dose: accessed at any time during the intervention period

- Implementation outcome: implementation of healthy eating practices

- Justification: the provision of support and resources via web-based programs is highly acceptable among ECEC staff and has been used within previous interventions within the ECEC setting [22,27,28].
- Feedback on behavior

- Feedback on outcome of behavior

- Self-monitoring of behavior

- Goal-setting (outcome and behavior)

- Action planning

- Problem solving

- Review goals (outcome and behavior)

- Demonstration of behavior

- Restructuring the physical environment

- $\quad$ Adding objects to the environment

- Prompts or cues

- Credible source

\section{Health promotion officer}


Mode of delivery and implementation Application of the implementation strategy according to Proctor [41] strategy according to ERIC $^{\mathrm{a}}$ [39]

Educational outreach visit

Identify and prepare a center champion

Mandate change

Ongoing consultation and local technical assistance
- $\quad$ Actor: $\mathrm{HPO}^{\mathrm{d}}$

- Action: 1.5-2-hour practical face-to-face training session with an HPO was provided to nominated supervisors and center champions to introduce the web-based program and support implementation of the healthy eating practices.

- Target: nominated supervisors and center champion knowledge and ability to implement change

- Temporality: one-off face-to-face training session (1.5-2 hours) at the start of the intervention (2-8 weeks after baseline)

- Dose: one-off training session

- Implementation outcome: adoption of the intervention

- Justification: face-to-face training within previous ECEC-based interventions has been highly acceptable and used within previous interventions conducted by the research team $[27,42]$

- Actor: center champion

- Action: center nominated supervisors were asked to identify and prepare a staff member who could dedicate themselves to endorsing and driving implementation of the intervention within their center and asked to attend the educational outreach visit.

- Target: center champions; staff investment and motivation to change, formalized guidance and demonstrated support for staff

- Temporality: commencement of the intervention period

- Dose: ongoing endorsement and support for use of the web-based program throughout the intervention period

- Implementation outcome: adoption of the intervention and implementation of healthy eating practices

- Justification: preparing a champion has been identified as an effective strategy to drive implementation and has been used in previous trials by the research team $[39,43,44]$.

- Actor: HPO, nominated supervisor, and center champion

- Action: an $\mathrm{MoU}^{\mathrm{e}}$ was developed to outline the responsibilities and level of commitment expected from both the center and the HPO in working to implement the targeted healthy eating practices. Center nominated supervisors and champions discussed the MoU with the HPO and tailored the content of the MoU to suit the needs of the center.

- Target: nominated supervisors and center champion investment and motivation to change, formalized guidance and demonstrated support for staff

- Temporality: MoU drafted during the face-to-face educational outreach visit and finalized and signed by the nominated supervisor, center champion, and HPO 2 weeks following the training

- Dose: one-off MoU during the face-to-face educational outreach visit, followed by ongoing advocating and support for use of the web-based program by the nominated supervisor and center champion to center staff during the intervention period

- Implementation outcome: adoption of the intervention

- Justification: securing executive support from nominated supervisors has been effective in improving implementation of healthy eating practices in previous ECEC-based interventions [19]
Behavior change technique actioned via the implementation strategy

- Instruction on how to perform behavior

- Demonstration on how to perform behavior

- Identification of self as role model

- Social support (unspecified)

- Commitment

- Social support (unspecified)

- Social support (unspecified)

- Verbal persuasion about capability 


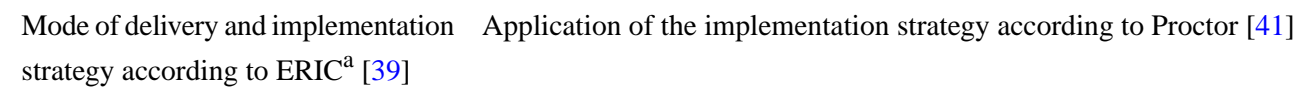

Behavior change technique actioned via the implementation strategy

- Actor: HPO

- Action: a telephone call was provided to nominated supervisors and center champions to discuss barriers to center implementation of healthy eating practices and the use of the Childcare EATS program, and to develop strategies to address such barriers. Email and telephone support was provided by HPOs upon center request.

- Target: nominated supervisors and center champion prioritization and confidence to implement change, formalized guidance, and support

- Temporality: 1 telephone call made to centers approximately 2 months following the face-to-face training session

- Dose: once during the intervention period

- Implementation outcome: adoption of the intervention and implementation of healthy eating practices

- Justification: ongoing consultation has been shown to be effective in improving implementation, staff motivation and problem solving within ECEC-based interventions [45,46].

${ }^{a}$ ERIC: expert recommendations for implementing change.

${ }^{b}$ EATS: Electronic Assessment Tool and Support.

${ }^{c}$ ECEC: early childhood education and care.

${ }^{\mathrm{d}} \mathrm{HPO}$ : health promotional officers.

${ }^{\mathrm{e}} \mathrm{MoU}$ : memorandum of understanding.

\section{Control}

Centers allocated to the control group received usual care during the intervention period, including general support from HPOs external to the research team upon request to implement the NSW state obesity-prevention program (ie, Munch \& Move). The provision of such support was centrally monitored by the research team, with 1 center receiving educational materials to support the implementation of healthy eating and physical activity practices before baseline data collection.

\section{Data Collection and Measures}

Baseline data were collected between September 2019 and December 2019, and follow-up data were collected between September 2020 and October 2020. A summary of the study outcomes and time points of measurement is provided in Table 2 .

Table 2. Study outcomes and time points of measurement.

\begin{tabular}{ll}
\hline Study outcome & Time points of measurement \\
\hline Center and child demographics & Baseline \\
Feasibility of the evaluation procedures & Baseline \\
$\quad$ Childcare center and child consent rates & Baseline \\
$\quad$ Completion of data collection components & \\
Uptake, acceptability, and appropriateness of the intervention and implementation strategies & 6 months \\
$\quad \begin{array}{l}\text { Delivery of the implementation strategies } \\
\text { Engagement with the Childcare EATS }{ }^{\text {a }} \text { web-based program }\end{array}$ & 6 months \\
$\quad$ Acceptability of the implementation strategies & 12 -month follow-up \\
$\quad$ Appropriateness of the intervention & 12 -month follow-up \\
Cost of implementation strategy delivery & Continuously across study period \\
Implementation of targeted healthy eating practices within the intervention group & Baseline and 6 months \\
\hline
\end{tabular}

${ }^{\mathrm{a}}$ EATS: Electronic Assessment Tool and Support.

\section{Outcomes: Center and Child Demographics}

At baseline, a web-based or telephone interview (depending on center preference) with center nominated supervisors was conducted to collect center demographic information, including the type of center (ie, preschool or long day care), center operating hours, number of Aboriginal or Torres Strait Islander enrollments, and number of children enrolled aged between 2 and 5 years. Center area SES and geographic location were determined using the center postcodes. Nominated supervisor 
demographic information, including age, was also collected during the baseline interviews. A web-based or telephone interview (depending on center preference) was conducted with center champions at follow-up to collect demographic information, including age.

Information recorded on parent consent forms was used to examine the child demographics. Parents reported the child's age, sex (as recorded on the child's birth certificate), Aboriginal or Torres Strait Islander background, and usual number of days attending care.

\section{Feasibility of the Evaluation Procedures}

The feasibility of the evaluation procedures, defined as the extent to which the research can be effectively carried out within the ECEC setting [47], was assessed via parent and center consent rates and completion of data collection components.

Childcare center and child consent rates were assessed using internal records kept by the research team, center, and child consent forms. Center consent rates were calculated as the number of consenting centers divided by the number of eligible centers that were approached to participate in the study. Reasons for centers declining to participate and ineligibility were recorded by the staff member conducting the recruitment telephone calls. Research assistants present on the days of data collection collated all returned child consent forms, including those from parents who did not provide consent for their child to participate in the study. Class lists specific to the days of data collection were obtained from each participating center to determine the total number of eligible children, with consent rates calculated as the number of consenting children divided by the total number of eligible children.

Completion of data collection components including lunchbox observations and measurements, web-based or telephone interviews with nominated supervisors, and observations of the center nutrition environments, was monitored via internal records kept by the research team. These data collection components were used to evaluate the originally planned trial outcomes related to the center nutrition environment and child dietary intake. Center completion of each individual component of data collection (web-based or telephone interview and assessment of center nutrition environments) was collated and entered into a tracking spreadsheet by a member of the research team. The number of complete child dietary intake data collection forms completed during center site visits was counted and included in the tracking spreadsheet.

\section{Uptake, Acceptability, and Appropriateness of the Intervention and Implementation Strategies}

The delivery of the implementation strategies was monitored using internal records maintained by the research team. For each center, the following information was recorded: center receipt of each implementation strategy (ie, number of centers that were offered and accepted or declined each strategy), date, duration, and type (ie, email, telephone, or face-to-face) of each implementation strategy delivered, the role of center staff receiving the implementation strategy (ie, nominated supervisor or center champion), and the delivery of BCTs within each implementation strategy (Table 1).
Engagement with the Childcare EATS web-based program was assessed via Google Analytics [48] embedded within the program. Information collected via the analytics included center completion of self-assessments (ie, audit with feedback), development of action plans (ie, developing a formal implementation blueprint), frequency of centers accessing educational materials, total log-ins to Childcare EATS, and average duration of the log-ins. Such measures have been reported in previous ECEC web-based interventions [27,49].

The acceptability of the implementation strategies, defined as the perception among center staff that the implementation strategies are satisfactory, palatable, or agreeable [47], was assessed through web-based and telephone interviews with nominated supervisors and center champions at follow-up. Interview items were modified from those developed by Weiner et al [50] and those used by the research team in previous ECEC-based studies [27,51]. In total, 10 items captured information on the perceived effectiveness (eg, ease of use and helpful in assessing and improving implementation of practices) of the Childcare EATS web-based program and usefulness of the implementation support strategies [27,47,51]. Nominated supervisors responded to each item on a 5-point Likert scale (1=strongly agree to $5=$ strongly disagree), with the proportion reporting 2 or lower (agree and strongly agree) for each item calculated.

The appropriateness of the intervention, defined as the perceived fit, relevance, or compatibility of the intervention and for the childcare setting [50], was assessed during the web-based or telephone interview with nominated supervisors at follow-up. In total, 4 items captured information on the perceived fit and suitability of healthy eating practices, using modified items by Weiner et al [50]. Nominated supervisors responded to each item on a 5-point Likert scale (1=strongly agree to 5=strongly disagree), with the proportion reporting 2 or lower (agree and strongly agree) for each item calculated.

\section{Cost to Deliver and Receive Implementation Strategies}

The direct cost of each implementation strategy delivered by HPOs, including labor (ie, HPO preparation, administration, and delivery of the strategy) and travel, was calculated. Service delivery costs were recorded by the HPOs delivering the intervention. Costs (in Aus \$ and US \$, 2019/2020) were calculated by multiplying the time spent (in hours) on each implementation strategy by the hourly wage rate of HPOs delivering the intervention. The cost for nominated supervisors and center champions to receive the implementation strategies delivered by HPOs and embedded within the web-based program was also calculated. Data to calculate center costs were recorded by the HPOs delivering the intervention in addition to the time spent in the web-based program captured by the analytics data. Similar to previous studies examining the cost of receiving interventions within the childcare setting [23], costs were calculated by multiplying the time spent (in hours) receiving each implementation strategy by the estimated hourly wage rate of nominated supervisors and educators [52]. 


\section{Implementation of Targeted Healthy Eating Practices Within the Intervention Group}

Self-reported implementation of the 5 targeted healthy eating practices within the intervention group was assessed via baseline nominated supervisor interview data and self-assessments completed by centers via the web-based program at any time point throughout the intervention. In total, 26 items were based on the validated Environment and Policy Assessment and Observation Self-Report [53] and the tool developed by Dodds et al [54] were used to measure the implementation of the 5 healthy eating practices.

In addition, we also assessed contextual factors influencing the center implementation of healthy eating practices, assessed through web-based and telephone interviews with nominated supervisors at follow-up. A total of 5 interview items were based on constructs within 3 of the 5 domains of the Consolidated Framework for Implementation Research (inner setting: compatibility with center values and direction), innovation characteristics (perceived complexity and cost), and outer setting (external influences such as policies and regulations) to identify factors associated with implementation [55]. Nominated supervisors responded to each item on a 5-point Likert scale ( $1=$ strongly agree to $5=$ strongly disagree), with the proportion reporting 2 or lower (agree and strongly agree) for each item calculated.

\section{Statistical Analysis}

All statistical analyses were performed using STATA v14 (StataCorp LLC) [56]. All data were analyzed using descriptive statistics. Chi-square analyses were used to compare characteristics of consenting and nonconsenting centers as well as center and child characteristics between the intervention and control groups at baseline. Center locality was classified as either urban (ie, major cities) or rural (ie, inner regional, outer regional, and remote) according to the Australian Statistical Geography Standard [57]. The 2016 Socio-Economic Indexes for Areas was used to classify centers as being located in the least disadvantaged (high SES) or most disadvantaged (low SES) areas [37]. Center postcodes ranked in the top 50\% of NSW were classified as least disadvantaged and the lower 50\% of postcodes as the most disadvantaged.

\section{Results}

\section{Overview}

A total of 22 centers and 448 children participated in the study, with $11(50 \%)$ centers randomized to the intervention group and $11(50 \%)$ to the control group (see Figure 1 for the CONSORT diagram). The demographic characteristics of consenting centers and children are summarized in Table 3. There were no significant differences in center SES or center geographic location between the consenting and nonconsenting centers. In addition, there were no significant differences in the center or child characteristics between the intervention and control groups at baseline. 
Figure 1. Study flow diagram.

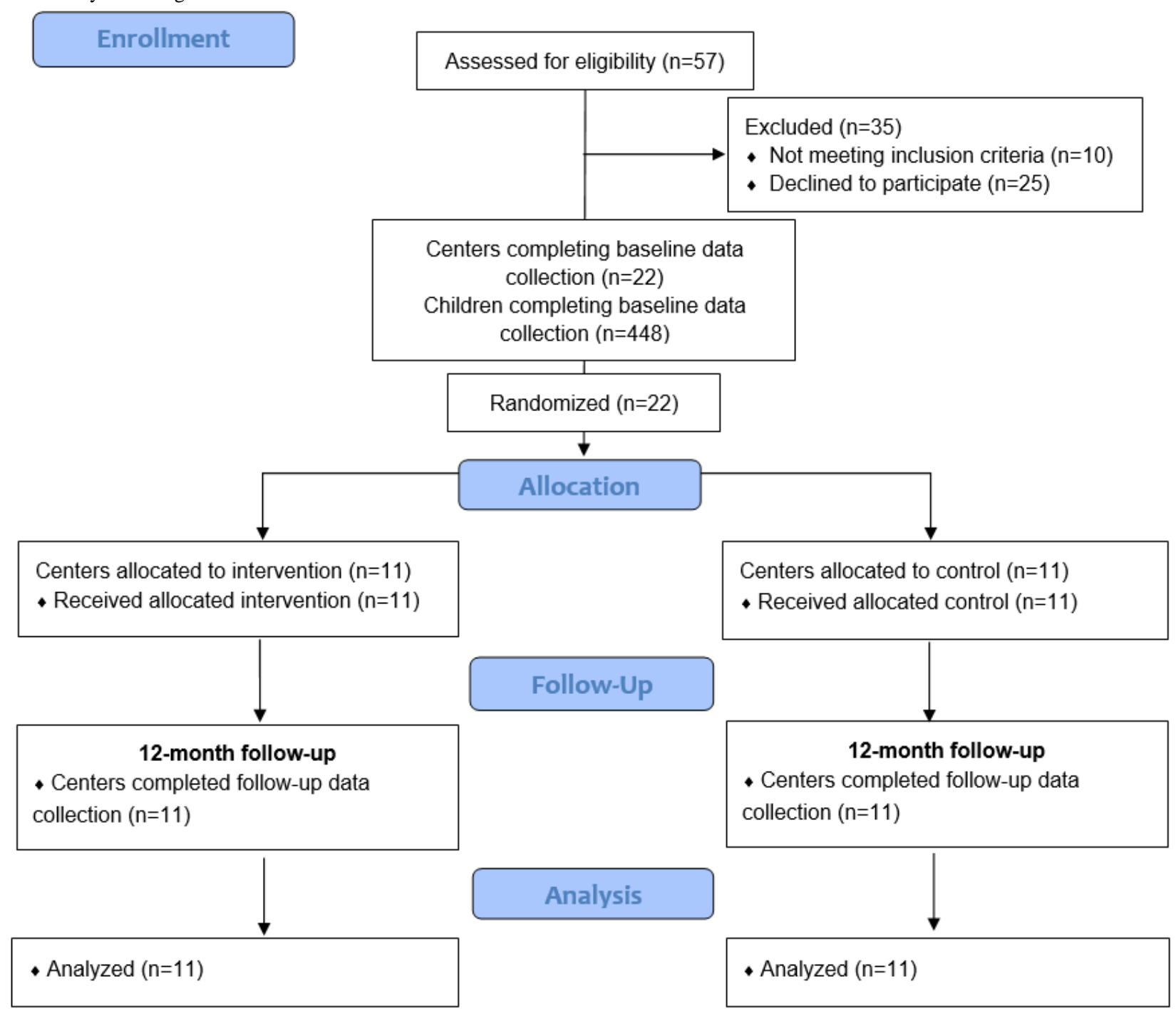


Table 3. Demographic characteristics of participating centers and children $(\mathrm{N}=11)$.

\begin{tabular}{|c|c|c|}
\hline Center characteristics & Intervention & Control \\
\hline \multicolumn{3}{|l|}{ Type of center } \\
\hline Preschool, n (\%) & $10(90)$ & $10(90)$ \\
\hline Long day care, n (\%) & $1(10)$ & $1(10)$ \\
\hline Child enrollments aged 2-5 years, mean (SD) & $30.73(11.24)$ & $29.0(8.63)$ \\
\hline Aboriginal child enrollments, mean (SD) & $5.0(4.58)$ & $4.64(3.32)$ \\
\hline \multicolumn{3}{|l|}{ SEIFA $^{\text {a,b }}$} \\
\hline Most disadvantaged (low SES $\left.{ }^{\mathrm{c}}\right), \mathrm{n}(\%)$ & $4(36)$ & $4(36)$ \\
\hline Least disadvantaged (high SES), n (\%) & $7(64)$ & $7(64)$ \\
\hline \multicolumn{3}{|l|}{ Geographic location } \\
\hline Urban (major cities), n (\%) & $8(73)$ & $8(73)$ \\
\hline Rural (inner regional, outer regional, and remote), n (\%) & $3(27)$ & $3(27)$ \\
\hline \multicolumn{3}{|l|}{ Nominated supervisor characteristics } \\
\hline Age, mean (SD) & $37.68(5.92)$ & $43.91(10.57)$ \\
\hline Center champion characteristics, $N$ & 6 & $-{ }^{\mathrm{d}}$ \\
\hline Age (years), mean (SD) & $44.17(6.40)$ & \\
\hline Child characteristics, $\mathbf{N}$ & 246 & 202 \\
\hline Age (years), mean (SD) & $4.68(0.66)$ & $4.65(0.68)$ \\
\hline Gender, $\mathbf{N}$ & 246 & 202 \\
\hline Female, $\mathrm{n}(\%)$ & $122(49.5)$ & $88(43.5)$ \\
\hline Male, n (\%) & $124(50.4)$ & $114(56.4)$ \\
\hline Children of Aboriginal and Torres Strait Islander background, $\mathrm{n}(\%)$ & $24(9.7)$ & $20(9.9)$ \\
\hline Days attending care, mean (SD) & $2.63(0.88)$ & $2.57(0.74)$ \\
\hline
\end{tabular}

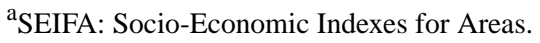

${ }^{\mathrm{b}}$ The 2016 Socio-Economic Indexes for Areas was used to classify centers as being located in the least disadvantaged (high socioeconomic status) or most disadvantaged (low socioeconomic status) areas. Center postcodes ranked in the top 50\% of New South Wales were classified as least disadvantaged and the lower $50 \%$ of postcodes as the most disadvantaged.

${ }^{\mathrm{c}} \mathrm{SES}$ : socioeconomic status.

${ }^{\mathrm{d}}$ Data not available (this item was only applied to nominated supervisors).

\section{Feasibility of the Evaluation Procedures}

\section{Childcare Center and Child Consent Rates}

Of the 85 potentially eligible centers within the sampling frame, $57(67 \%)$ centers were approached in random order to participate in the study. Of the 57 centers, $10(18 \%)$ centers were ineligible (NSW Department of Education center: 6/10, 60\%; involved in another healthy eating or physical activity research trial: $1 / 10$, $10 \%$; and provided food to children: $3 / 10,30 \%)$ and $25(44 \%)$ centers declined to participate (lack of time: $21 / 25,84 \%$; study of lessor importance: $2 / 25,8 \%$; and lack of staff capacity: $2 / 25$, $8 \%$ ). This resulted in an overall study consent rate of $47 \%$ $(22 / 57)$. No centers withdrew from the trial following randomization.

A potential 670 children were eligible to participate in the lunchbox measurements, of whom, 502 (74.9\%) provided consent to participate. The consent rate ranged from 53\% (16/30) to $96 \%(24 / 25)$ within the participating centers $(285 / 374,76.2 \%$ children within intervention centers and 217/296, $73.3 \%$ children within control centers).

\section{Completion of Data Collection Components}

Baseline lunchbox observations and measurements conducted to assess the impact of the intervention on child dietary intake were completed for $100 \%$ (448/448) of consenting children who were in attendance on data collection days at baseline. The remaining $10.8 \%(54 / 502)$ of the children were absent on the data collection days. Baseline observations of the nutrition environment and web-based or telephone interviews with center nominated supervisors were completed for $100 \%(22 / 22)$ of participating centers.

\section{Uptake, Acceptability, and Appropriateness of the Intervention and Implementation Strategies}

\section{Delivery of Implementation Strategies}

For implementation strategies delivered by the HPO, $100 \%$ of center nominated supervisors or directors were offered and 
received the educational outreach visit (ie, face-to-face training session) with the HPO at the commencement of the intervention. The mean duration of the educational outreach visit was 92.73 (SD 21.83) minutes. All centers $(n=11)$ were invited to nominate and prepare a staff member as center champion, with $55 \%(6 / 11)$ of centers nominating a staff member, and $83 \%(5 / 6)$ of these also attending the educational outreach visit. The memorandum of understanding (MoU; ie, mandate change) was drafted with all intervention centers $(\mathrm{n}=11)$, with a signed MoU returned by $55 \%(6 / 11)$ of the centers. Ongoing consultation and local technical assistance (ie, follow-up support call provided by the HPO) were offered to $100 \%(11 / 11)$ of the intervention centers, with $91 \%(10 / 11)$ of the centers accepting the call. The mean duration of the follow-up support call was 11.9 (SD 4.70) minutes.

For implementation strategies within the web-based program, overall, $100 \%(11 / 11)$ of centers were provided access to and undertook audit with feedback (ie, self-assessment), developed a formal implementation blueprint (ie, action plan), and accessed the educational materials via the Childcare EATS web-based program.

All intervention centers $(\mathrm{n}=11)$ received BCTs as intended in $57 \%$ (4/7) of the implementation strategies (Table 4). Additional BCTs (instruction on how to perform the behavior, problem solving, social support [practical], and action planning) were used within the ongoing consultation and local technical assistance strategy in $37 \%(4 / 11)$ of the centers owing to the HPO responding to the needs of the center and tailoring the advice accordingly. Low uptake of the mandate change and identification and preparation of center champion implementation strategies resulted in only 55\% (6/11) of the centers receiving the BCTs within these strategies. 
Table 4. Behavior change techniques delivered within implementation strategies $(\mathrm{N}=11)$.

\begin{tabular}{|c|c|}
\hline Mode of delivery, implementation strategy, and behavior change technique & Number of centers \\
\hline \multicolumn{2}{|l|}{ Web-based program } \\
\hline \multicolumn{2}{|l|}{ Audit with feedback } \\
\hline Feedback on behavior & $11(100)$ \\
\hline Feedback on outcome of behavior & $11(100)$ \\
\hline Self-monitoring of behavior & $11(100)$ \\
\hline \multicolumn{2}{|l|}{ Develop a formal implementation blueprint } \\
\hline Goal-setting (outcome and behavior) & $11(100)$ \\
\hline Action planning & $11(100)$ \\
\hline Problem solving & $11(100)$ \\
\hline Review goals (outcome and behavior) & $11(100)$ \\
\hline \multicolumn{2}{|l|}{ Distribute educational materials } \\
\hline Demonstration of behavior & $11(100)$ \\
\hline Restructuring the physical environment & $11(100)$ \\
\hline Adding objects to the environment & $11(100)$ \\
\hline Prompts or cues & $11(100)$ \\
\hline Credible source & $11(100)$ \\
\hline \multicolumn{2}{|l|}{ Health promotion officer } \\
\hline \multicolumn{2}{|l|}{ Educational outreach visit } \\
\hline Instruction on how to perform behavior & $11(100)$ \\
\hline Demonstration on how to perform behavior & $11(100)$ \\
\hline \multicolumn{2}{|l|}{ Ongoing consultation and local technical assistance } \\
\hline Social support (unspecified) & $10(91)$ \\
\hline Verbal persuasion about capability & $10(91)$ \\
\hline Instruction on how to perform behavior ${ }^{\mathrm{a}}$ & $3(27)$ \\
\hline Problem solving $^{\mathrm{a}}$ & $1(9)$ \\
\hline Social support (practical) ${ }^{\mathrm{a}}$ & $1(9)$ \\
\hline Action planning $^{\mathrm{a}}$ & $3(27)$ \\
\hline \multicolumn{2}{|l|}{ Mandate change } \\
\hline Commitment & $6(55)$ \\
\hline Social support (unspecified) & $6(55)$ \\
\hline \multicolumn{2}{|l|}{ Identify and prepare a center champion } \\
\hline Identification of self as role model & $6(55)$ \\
\hline Social support (unspecified) & $6(55)$ \\
\hline
\end{tabular}

${ }^{a}$ Additional behavior change techniques used within the ongoing consultation and local technical assistance implementation strategy beyond that specified in the intervention protocol.

\section{Engagement With the Web-Based Program}

The intervention center's engagement with the Childcare EATS web-based program is detailed in Table 5. At the 6-month follow-up, intervention centers had logged in to the program on an average of 5.18 (SD 2.52) times, spending an average of 19.90 (SD 11.21) minutes in the program per log-in. Centers completed an average of 2.90 (SD 2.02) self-assessments and developed an average of 2.09 (SD 1.30) action plans. A total of 6 staff members from 4 intervention centers completed web-based professional development accessible via the web-based program or the NSW state obesity-prevention program website (ie, Munch \& Move) during the intervention period compared with no staff members from control centers. 
Table 5. Center engagement with Childcare Electronic Assessment Tool and Support web-based program across 6 months.

\begin{tabular}{lll}
\hline Engagement & Value, mean $(\mathrm{SD})$ & Value, median (IQR) \\
\hline Total log-ins & $5.18(2.52)$ & $4.00(4.00-5.00)$ \\
$\begin{array}{l}\text { Average log-in duration (min- } \\
\text { utes) }\end{array}$ & $19.90(11.21)$ & $17.44(10.24-30.03)$ \\
Self-assessments completed & $2.90(2.02)$ & $2.00(1.00-4.00)$ \\
$\begin{array}{l}\text { Action plans developed } \\
\begin{array}{l}\text { Number of times educational } \\
\text { materials were accessed }\end{array}\end{array}$ & $12.09(1.30)$ & $2.00(1.00-3.00)$ \\
\hline
\end{tabular}

\section{Acceptability of the Intervention and Implementation Strategies}

The web-based program was reported to be an acceptable method for assessing healthy eating practices by most nominated supervisors $(10 / 11,91 \%)$ and center champions $(5 / 6,83 \%$;
Table 6). The implementation strategies provided by HPOs, including the educational outreach visit (ie, face-to-face training) and ongoing support (ie, support call), were considered to be acceptable by nominated supervisors $(10 / 11,91 \%$ to $11 / 11$, $100 \%)$. Acceptability of the implementation strategies was lower among center champions $(2 / 6,33 \%$ to $5 / 6,83 \%)$. 
Table 6. Acceptability and appropriateness of the web-based intervention and implementation strategies.

\begin{tabular}{|c|c|c|}
\hline Characteristics & $\begin{array}{l}\text { Nominated supervisors } \\
(\mathrm{n}=11), \mathrm{n}(\%)\end{array}$ & $\begin{array}{l}\text { Center champions } \\
(\mathrm{n}=6), \mathrm{n}(\%)\end{array}$ \\
\hline \multicolumn{3}{|l|}{ Measure (agree or strongly agree) } \\
\hline $\begin{array}{l}\text { Using the web-based program is an acceptable method for assessing if our service is } \\
\text { meeting the healthy eating policies and practices. }\end{array}$ & $10(91)$ & $5(83)$ \\
\hline $\begin{array}{l}\text { The web-based program was useful in my service to help meet the healthy eating policies } \\
\text { and practices. }\end{array}$ & $11(100)$ & $5(83)$ \\
\hline $\begin{array}{l}\text { Using the web-based program improved my service's performance in meeting the healthy } \\
\text { eating policies and practices. }\end{array}$ & $10(91)$ & $5(83)$ \\
\hline I would recommend the web-based program to other childcare services. & $10(91)$ & $5(83)$ \\
\hline $\begin{array}{l}\text { I intend to continue to use the web-based program to help our service meet the healthy } \\
\text { eating policies and practices. }\end{array}$ & $10(91)$ & $5(83)$ \\
\hline I thought the web-based program was easy to use. & $10(91)$ & $-{ }^{\mathrm{a}}$ \\
\hline \multicolumn{3}{|l|}{ Measure (useful or very useful) } \\
\hline I found the face-to-face training session (ie, educational outreach visit) useful. & $10(91)$ & $5(83)$ \\
\hline I found the garnering of managerial support (ie, mandate change) useful. & $11(100)$ & $2(33)$ \\
\hline $\begin{array}{l}\text { I found the ongoing telephone support (ie, ongoing consultation and local technical assis- } \\
\text { tance) provided by the health promotion officers useful. }\end{array}$ & $10(91)$ & $2(33)$ \\
\hline I found nominating a center champion (ie, identify and prepare a center champion) useful. ${ }^{\mathrm{b}}$ & $5(83)$ & - \\
\hline Appropriateness (agree or strongly agree) & $11(100)$ & - \\
\hline \multicolumn{3}{|l|}{ The healthy eating policies and practices seem fitting. } \\
\hline \multicolumn{3}{|l|}{ The healthy eating policies and practices seems suitable. } \\
\hline \multicolumn{3}{|l|}{ The healthy eating policies and practices seem applicable. } \\
\hline \multicolumn{3}{|l|}{ The healthy eating policies and practices seem like a good match. } \\
\hline \multicolumn{2}{|c|}{ Contextual factors influencing implementation of healthy eating practices (agree or strongly agree) } & - \\
\hline The healthy eating policies and practices are consistent with our center philosophy. & $10(91)$ & \\
\hline $\begin{array}{l}\text { The healthy eating policies and practices are consistent with the National Quality } \\
\text { Framework. }\end{array}$ & $10(91)$ & \\
\hline The healthy eating policies and practices are costly to implement. & $0(0)$ & \\
\hline The healthy eating policies and practices are difficult to implement. & $4(36)$ & \\
\hline Centers within our region would be supportive of the healthy eating policies and practices. & $10(91)$ & \\
\hline
\end{tabular}

${ }^{\mathrm{a}}$ Data not available (this item was only applied to nominated supervisors).

${ }^{b}$ This item was only applied to centers that nominated a center champion $(n=6)$.

\section{Appropriateness of the Intervention}

In total, $100 \%(11 / 11)$ of nominated supervisors within the intervention group agreed or strongly agreed that healthy eating policies and practices seem fitting, suitable, applicable, and a good match (Table 6).

\section{Cost to Deliver and Receive Implementation Strategies}

The total cost to the health service for the HPO to deliver the implementation strategies (ie, educational outreach visit, mandate change, and ongoing consultation) was Aus \$1351.25 (US \$972.64), average per center: Aus \$ 122.84 (US \$88.42). Overall, the educational outreach visits cost a total of Aus \$ 1143.08 (US \$822.79), average per center: Aus \$ 103.92 (US $\$ 74.80)$, including travel to the center and follow-up correspondence with center staff; mandate change cost a total of Aus \$ 43.44 (US \$31.27), average per center: Aus \$ 3.95 (US $\$ 2.84)$; and ongoing consultation cost a total of Aus $\$ 164.73$ (US \$118.57), average per center: Aus \$ 14.98 (US \$10.78). The total cost to centers for nominated supervisors and center champions to receive all implementation strategies (ie, those delivered by the HPO and embedded within the web-based program) was Aus \$ 1516.40 (US \$1091.51), average per center: Aus \$137.85 (US \$99.23). The cost to receive the implementation strategies delivered by the HPO was Aus \$ 1052.29 (US \$757.44), average per center: Aus \$ 95.66 (US $\$ 68.86$ ), whereas the cost to receive the implementation strategies embedded within the web-based program was Aus \$ 464.11 (US \$334.07), average per center: Aus \$ 42.19 (US \$30.37). 
Implementation of Targeted Healthy Eating Practices Within the Intervention Group

The proportion of centers implementing targeted healthy eating practices improved in 4 of the 5 practices from baseline to follow-up (Table 7). The greatest improvement was reported in center educator use of feeding practices that support children's healthy eating, increasing from $18 \%(2 / 11)$ to $82 \%(9 / 11)$. The proportion of centers supporting families to provide healthier foods consistent with dietary guidelines decreased from $82 \%$ (9/11) to 55\% (6/11). At follow-up, $18 \%$ (2/11) of centers were implementing all 5 healthy eating practices, whereas none were at baseline. The mean number of practices implemented per center increased from 3.36 (SD 1.21) at baseline to 4.36 (SD 1.21) at follow-up. When examining the change in practice implementation between the most (low SES) and least (high SES) disadvantaged centers, the number of most disadvantaged centers supporting families to provide healthier foods consistent with dietary guidelines reduced from $100 \%$ (4/4) at baseline to $25 \%(1 / 4)$ at follow-up compared with no change in least disadvantaged centers (Table 8).

In total, 91\% (10/11) of nominated supervisors reported that healthy eating practices were consistent with the philosophy of their service and consistent with the ECEC settings regulatory standards (ie, the National Quality Framework; Table 6).

Table 7. Intervention group implementation of healthy eating practices $(\mathrm{N}=11)$.

\begin{tabular}{|c|c|c|c|}
\hline Healthy eating practice & $\begin{array}{l}\text { Centers implementing at baseline, } \\
\mathrm{n}(\%)\end{array}$ & $\begin{array}{l}\text { Centers implementing at follow-up, } \\
\mathrm{n}(\%)\end{array}$ & Change, $\mathrm{n}(\%)$ \\
\hline Provision of intentional healthy eating learning experiences & $4(36)$ & $6(55)$ & $2(18)$ \\
\hline $\begin{array}{l}\text { Comprehensive written nutrition policy that outlines key } \\
\text { healthy eating practices }\end{array}$ & $8(73)$ & $10(91)$ & $2(18)$ \\
\hline $\begin{array}{l}\text { Staff participating in professional development targeting } \\
\text { healthy eating }\end{array}$ & $3(27)$ & $6(55)$ & $3(27)$ \\
\hline $\begin{array}{l}\text { Educator use of feeding practices that support children's } \\
\text { healthy eating }\end{array}$ & $2(18)$ & $9(82)$ & $7(64)$ \\
\hline $\begin{array}{l}\text { Supporting families to provide healthier foods consistent } \\
\text { with dietary guidelines }\end{array}$ & $9(82)$ & $6(55)$ & $-3(27)$ \\
\hline
\end{tabular}

Table 8. Intervention group implementation of healthy eating practices by Socio-Economic Indexes for Areas classification $(\mathrm{N}=1)^{\mathrm{a}}$.

\begin{tabular}{|c|c|c|c|c|c|c|}
\hline \multirow[t]{2}{*}{ Healthy eating practice } & \multicolumn{3}{|l|}{ Low $\operatorname{SES}^{\mathrm{b}}(\mathrm{n}=4), \mathrm{n}(\%)$} & \multicolumn{3}{|l|}{ High SES (n=7), n (\%) } \\
\hline & $\begin{array}{l}\text { Most disadvantaged } \\
\text { centers implementing } \\
\text { at baseline }\end{array}$ & $\begin{array}{l}\text { Most disadvantaged } \\
\text { centers implementing } \\
\text { at follow-up }\end{array}$ & Change & $\begin{array}{l}\text { Least disadvantaged } \\
\text { centers implementing } \\
\text { at baseline }\end{array}$ & $\begin{array}{l}\text { Least disadvantaged } \\
\text { centers implementing } \\
\text { at follow-up }\end{array}$ & Change \\
\hline $\begin{array}{l}\text { Provision of intentional } \\
\text { healthy eating learning } \\
\text { experiences }\end{array}$ & $2(50)$ & $2(50)$ & $0(0)$ & $2(29)$ & $4(57)$ & $2(29)$ \\
\hline $\begin{array}{l}\text { Comprehensive written } \\
\text { nutrition policy that } \\
\text { outlines key healthy } \\
\text { eating practices }\end{array}$ & $3(75)$ & $3(75)$ & $0(0)$ & $5(71)$ & $7(100)$ & $2(29)$ \\
\hline $\begin{array}{l}\text { Staff participating in } \\
\text { professional develop- } \\
\text { ment targeting healthy } \\
\text { eating }\end{array}$ & $1(25)$ & $1(25)$ & $0(0)$ & $1(14)$ & $5(71)$ & $4(57)$ \\
\hline $\begin{array}{l}\text { Educator use of feeding } \\
\text { practices that support } \\
\text { children's healthy eat- } \\
\text { ing }\end{array}$ & $1(25)$ & $4(100)$ & $3(75)$ & $1(14)$ & $5(71)$ & $4(57)$ \\
\hline $\begin{array}{l}\text { Supporting families to } \\
\text { provide healthier foods } \\
\text { consistent with dietary } \\
\text { guidelines }\end{array}$ & $4(100)$ & $1(25)$ & $-3(75)$ & $5(71)$ & $5(71)$ & $0(0)$ \\
\hline
\end{tabular}

${ }^{\mathrm{a}}$ The 2016 Socio-Economic Indexes for Areas was used to classify centers as being located in the least disadvantaged (high socioeconomic status) or most disadvantaged (low socioeconomic status) areas. Center postcodes ranked in the top 50\% of New South Wales were classified as least disadvantaged and the lower $50 \%$ of postcodes as the most disadvantaged.

${ }^{b}$ SES: socioeconomic status. 


\section{Discussion}

\section{Principal Findings}

This study aimed to assess the potential feasibility of a pilot cluster RCT of a web-based healthy eating implementation intervention in ECEC centers to undertake a fully powered implementation trial. The study also examined the uptake, acceptability, appropriateness, and actual cost of delivering the intervention and implementation strategies. Overall, the study findings indicate that the web-based intervention and most implementation strategies are highly feasible, low-cost, and acceptable to childcare center staff and can improve the implementation of healthy eating practices in ECEC centers.

The study obtained a high overall parental consent rate of $74.9 \%$ $(502 / 670)$ for children to participate in lunchbox measurements. However, the variability in parental consent across centers (ranging from $16 / 30,53 \%$ to $24 / 25,96 \%$ ) is worth noting. This variation may be owing to the differing relationships within centers between staff and parents regarding the contents of children's lunchboxes with previous studies reporting a reluctance from staff to communicate with parents in fear of having difficult conversations [58,59]. As such, some parents may have been reluctant to consent to lunchbox measurements owing to perceived judgment [58,59]. Although not dissimilar to previous web-based studies conducted within the ECEC setting, the overall study consent rate among centers was moderate at $47 \%[27,60,61]$. Similar to previous studies, barriers to center participation reported by staff included a lack of time and competing priorities [62]. As this study attempted to address such barriers by embedding the intervention within usual center processes (ie, aligning with ECEC accreditation standards), further consideration needs to be taken to better promote the intervention by aligning with current center priorities during study recruitment. However, once consented to the trial, the study data collection components were highly feasible, with $100 \%$ of participating centers completing child lunchbox measurements, center nutrition environment observations, and interviews with nominated supervisors. This indicates that such methods should be retained for a fully powered implementation trial.

Promising levels of uptake and acceptability of the implementation strategies used in this study were observed. The level of engagement with the web-based program was consistent with recommendations for centers to complete the self-assessment (audit with feedback) and develop action plans (formal implementation blueprint) twice during the intervention period. Such findings suggest that centers are likely to receive the intended dose of the intervention with the current implementation strategies. The promising levels of engagement may be attributed to the web-based program being easy to use as reported by nominated supervisors and aligned with usual center processes [63]. However, large SDs and wide IQRs for the number of log-ins and log-in duration indicate high variability in engagement with the web-based program across centers. Despite such variability being consistent with previous studies within the ECEC setting that used web-based modalities [27], exploration is needed to better understand the reasons behind the relatively lower levels of engagement for some centers.

As the intervention was largely delivered remotely, the overall cost to deliver the implementation strategies was minimal (total of Aus \$ 1351.25 [US \$ 931.88]; average per center: Aus \$ 122.84 [US \$ 86]). Therefore, the web-based intervention may be considered a low-cost alternative to support center implementation compared with traditional, highly intensive modalities. However, the study was unable to capture the costs associated with center staff implementing healthy eating practices, including the time spent disseminating information to parents. As such, future studies should consider conducting a cost-effective analysis, while capturing costs associated with center implementation of practices, to enable researchers, practitioners, funding bodies, and centers to determine whether investment in the web-based intervention produced an acceptable return and is a cost-effective approach to support the implementation of healthy eating practices at scale. Consistent with previous studies conducted within the ECEC setting [51,64], high levels of uptake and acceptability were found for most implementation strategies provided by HPOs, particularly the educational outreach visit $(11 / 11,100 \%)$ and local technical assistance $(10 / 11,91 \%)$. Despite previous literature suggesting that implementation strategies such as the MoU and center champions are useful for facilitating the uptake of interventions $[19,39,44]$, the relatively low uptake of these strategies is worth exploring. Although there was high acceptability of the center champion strategy in centers that nominated a champion $(9 / 10$, $83 \%$ ), a potential explanation for the lower uptake of the strategy may be the differing organizational structures within centers. Anecdotally, the uptake of center champions was higher in larger centers with greater staffing numbers and child enrollments, where the nominated supervisor often engages educational leads. The educational lead takes on additional advocacy roles among staff, lending them to the role of the center champion. In smaller centers however, the nominated supervisors often work as the educational lead themselves, acting as the main advocate among center staff. Therefore, the research team should consider alternative strategies, such as a local consensus approach [51] (ie, the entire center), to ensure that the uptake of the intervention remains high in centers where a sole champion is not a feasible strategy.

The improvement in implementation of 4 of the 5 targeted healthy eating practices within the intervention group is promising, with effect sizes ranging from $19 \%(2 / 11)$ to $64 \%$ (7/11). Such effect sizes are encouraging when compared with previous studies aimed at improving the implementation of practices within the ECEC setting [13]. A recent Cochrane systematic review, which examined the effectiveness of strategies aimed at improving the implementation of healthy eating and physical activity policies and practices, reported effect sizes as low as $2.5 \%$ [13]. Therefore, our findings show great promise for testing in a fully powered implementation trial. However, a decrease in centers supporting families to provide healthier foods consistent with dietary guidelines, particularly in those centers classified as most disadvantaged, is worth noting given this practice had the highest rates of implementation at baseline. A potential explanation for this 
reduction may be the competing information relating to COVID-19 distributed to parents during the intervention period (eg, communication regarding center safety protocols and changes to child attendance fees), resulting in support for parents to provide foods consistent with sector dietary guidelines being of lesser priority at this time. Research suggests a lack of skills, knowledge, and confidence in communicating with parents regarding healthy eating $[58,59,65]$ may also negatively impact the implementation of this practice. Using strategies, such as ongoing professional development, coaching, and training, have been suggested in recent studies to address such barriers and support ECEC staff to engage in positive and effective communication with parents [65]. As centers were encouraged to distribute the healthy eating resources to parents via usual communication methods (eg, parent communication apps, email, and written information), further consideration of the most effective method to facilitate staff communication with parents regarding healthy eating and nutrition may be required. Although the Childcare EATS engagement data provided important insights into the center use of the web-based program, the methods used and the reach of the center distribution of healthy eating information and resources (eg, number of parents who received the resources) could not be measured. In addition, we were unable to assess whether parents within the intervention group communicated healthy eating information provided by the center staff to other parents. There was a notable contrast in the implementation of this practice between centers classified as most and least disadvantaged. This contrast may potentially be explained by COVID-19 related impacts on resourcing (eg, staffing, budget, and time) within disadvantaged centers, who may have already been experiencing limited resources before the pandemic. A better understanding of the barriers faced by centers classified as most disadvantaged in communicating with parents should be sought to enable the development of appropriate strategies to support implementation of this practice. However, given the small sample size in this study, this finding is highly exploratory and should be interpreted with caution. In addition, collecting contextual data from parents regarding their preferred method of receiving healthy eating information from centers may also provide guidance on the most effective way to support parents in packing healthy lunchboxes for children to consume in care.
The findings from this study provide compelling data to support the conduct of a fully powered implementation trial. Importantly, despite the relatively low level of support provided to childcare centers to use the program, the level of engagement with the web-based program was relatively high, and large changes in practice implementation were observed. Findings from this study suggest that several improvements could be made to the intervention, including considering the appropriateness of the MoU and center champion and using strategies to support ECEC center staff engagement with parents regarding healthy eating. Finally, the inclusion of a nested evaluation within a future trial to assess the impact of the web-based intervention on individual-level outcomes, including child dietary intake and parent lunchbox packing practices, should be considered to gain greater insight into the effectiveness of the intervention beyond center-level outcomes.

\section{Limitations}

Although unavoidable because of restrictions relating to the COVID-19 pandemic, the inability to assess center nutrition environments and conduct child lunchbox assessments via direct observation to assess child-level outcomes as originally intended is a limitation of the study. In addition, although the data regarding the impact of the intervention on center implementation are promising, these data were only able to be collected within intervention centers with no comparison to the control group, and as such, should be interpreted with caution. Finally, as the study was conducted within 1 region of NSW, the generalizability of the findings beyond the region may be limited.

\section{Conclusions}

This pilot study provides compelling data to support the conduct of a larger trial assessing the impact of the web-based intervention on ECEC center implementation of healthy eating practices. The findings of this pilot study indicate that the web-based intervention is highly feasible, acceptable, appropriate, and low-cost. As this study is one of few examining the potential impact of a web-based intervention within the ECEC setting, a fully powered implementation trial is warranted to establish the true effects and examine the impact of the intervention at scale.

\section{Acknowledgments}

The authors wish to thank the staff at Hunter New England (HNE) Population Health, Population Health Aboriginal Advisory Network Group, and Christophe Lecathelinais for their statistical support. The authors would also like to thank the participating center staff, parents, and children. Finally, the authors would like to thank Kasia Bolsewicz, Melody Ng and Hannah Lamont for their contribution to the planning, implementation, and evaluation of the study. This project was partly funded by the Hunter Children's Research Foundation, Priority Research Center for Health Behavior University of Newcastle, Hunter Cancer Research Alliance, HNE Local Health District Grant, and Hunter Medical Research Institute. The funders played no role in conducting the trial. HNE Population Health and the University of Newcastle provided infrastructure and in-kind funding. CB receives salary support from a cofunded industry scholarship between HNE Population Health and the University of Newcastle. SLY receives salary support from the Australia Research Council Discovery Early Career Research Awards (DE170100382). LW receives salary support from the National Health Medical Research Council Career Development Fellowship (grant APP1128348), Heart Foundation Future Leader Fellowship (grant 101175), and a HNE Clinical Research Fellowship. The contents of this manuscript are the responsibility of the authors and do not reflect the views of Hunter Children's Research Foundation, Hunter Cancer Research Alliance, Priority Research Center for Health Behavior, Hunter Medical Research Institute Australian Research Council Heart Foundation, HNE Local Health District, or National Health Medical Research Council. 


\section{Authors' Contributions}

CB drafted the manuscript. SLY, AG, CB, NN, and LW conceived and designed the research and interventions. CB, AG, and JK delivered the intervention. $\mathrm{CB}, \mathrm{AG}, \mathrm{TW}$, and $\mathrm{JK}$ developed the evaluation protocols and led to the acquisition of data. $\mathrm{CB}$ conducted the data analyses. All authors contributed to drafting and final approval of the manuscript.

\section{Conflicts of Interest}

Authors CB, LW, AG, TW, and JK received salary support from Hunter New England Local Health District, which provided infrastructure and funding for the study.

\section{Multimedia Appendix 1}

CONSORT-eHEALTH checklist (V 1.6.1).

[PDF File (Adobe PDF File), 2954 KB-Multimedia Appendix 1]

\section{References}

1. GBD 2017 Diet Collaborators. Health effects of dietary risks in 195 countries, 1990-2017: a systematic analysis for the Global Burden of Disease Study 2017. Lancet 2019 May 11;393(10184):1958-1972 [FREE Full text] [doi: 10.1016/S0140-6736(19)30041-8] [Medline: 30954305]

2. Increasing fruit and vegetable consumption to reduce the risk of noncommunicable diseases . World Health Organization. URL: https://www.who.int/elena/titles/fruit vegetables ncds/en/ [accessed 2021-11-27]

3. Child care and early childhood education in Australia. Australian Institute of Family Studies. 2015. URL: https://aifs.gov.au/ publications/child-care-and-early-childhood-education-australia [accessed 2021-11-27]

4. Fox MK, Gearan E, Cannon J, Briefel R, Deming DM, Eldridge AL, et al. Usual food intakes of 2- and 3-year old U.S. children are not consistent with dietary guidelines. BMC Nutr 2016 Nov 15;2(1):67. [doi: 10.1186/s40795-016-0106-2]

5. NDNS: results from Years 5 and 6 (combined). GOV.UK. URL: https://www.gov.uk/government/statistics/ ndns-results-from-years-5-and-6-combined [accessed 2021-11-27]

6. Craigie AM, Lake AA, Kelly SA, Adamson AJ, Mathers JC. Tracking of obesity-related behaviours from childhood to adulthood: a systematic review. Maturitas 2011 Nov;70(3):266-284. [doi: 10.1016/j.maturitas.2011.08.005] [Medline: 21920682]

7. Population-based approaches to childhood obesity prevention. World Health Organization. URL: http://apps.who.int/iris/ bitstream/handle/10665/80149/9789241504782 eng.pdf?sequence=1 [accessed 2021-11-27]

8. Recommendations and public health and policy implications. World Cancer Research Fund International. URL: https:/ /www.wcrf.org/wp-content/uploads/2021/01/Recommendations.pdf [accessed 2021-11-27]

9. Hesketh KR, Griffin SJ, van Sluijs EM. UK Preschool-aged children's physical activity levels in childcare and at home: a cross-sectional exploration. Int J Behav Nutr Phys Act 2015 Sep 26;12:123 [FREE Full text] [doi: 10.1186/s12966-015-0286-1] [Medline: 26410252]

10. Childhood education and care, Australia. Australian Bureau of Statistics. URL: https://www.abs.gov.au/statistics/people/ education/childhood-education-and-care-australia/latest-release [accessed 2021-11-27]

11. Swinburn B, Malakellis M, Moodie M, Waters E, Gibbs L, Millar L, et al. Large reductions in child overweight and obesity in intervention and comparison communities 3 years after a community project. Pediatr Obes 2014 Dec;9(6):455-462. [doi: 10.1111/j.2047-6310.2013.00201.x] [Medline: 24203373]

12. Matwiejczyk L, Mehta K, Scott J, Tonkin E, Coveney J. Characteristics of effective interventions promoting healthy eating for pre-schoolers in childcare settings: an umbrella review. Nutrients 2018 Mar 01;10(3):293 [FREE Full text] [doi: 10.3390/nu10030293] [Medline: 29494537]

13. Wolfenden L, Barnes C, Jones J, Finch M, Wyse RJ, Kingsland M, et al. Strategies to improve the implementation of healthy eating, physical activity and obesity prevention policies, practices or programmes within childcare services. Cochrane Database Syst Rev 2020 Feb 10;2:CD011779 [FRE Full text] [doi: 10.1002/14651858.CD011779.pub3] [Medline: 32036618]

14. Stacey FG, Finch M, Wolfenden L, Grady A, Jessop K, Wedesweiler T, et al. Evidence of the potential effectiveness of centre-based childcare policies and practices on child diet and physical activity: consolidating evidence from systematic reviews of intervention trials and observational studies. Curr Nutr Rep 2017 Jul 22;6(3):228-246. [doi: $10.1007 / \mathrm{s} 13668-017-0212-\mathrm{z}]$

15. Benjamin Neelon SE, Briley ME, American Dietetic Association. Position of the American Dietetic Association: benchmarks for nutrition in child care. J Am Diet Assoc 2011 Apr;111(4):607-615. [doi: 10.1016/j.jada.2011.02.016] [Medline: 21443997]

16. Australian Govrenment Department of Health. URL: https://www.health.gov.au/resources/collections/ get-up-grow-resource-collection?utm source=health.gov. au\&utm medium=callout-auto-custom\&utm campaign=digital transformation [accessed 2021-11-27]

17. Munch \& Move. NSW Government. URL: https://healthykids.nsw.gov.au/ [accessed 2021-11-28] 
18. Kelly B, Hardy L, Howlett S, King L, Farrell L, Hattersley L. Opening up Australian preschoolers' lunchboxes. Aust N Z J Public Health 2010 Jun;34(3):288-292. [doi: 10.1111/j.1753-6405.2010.00528.x] [Medline: 20618271]

19. Bell AC, Davies L, Finch M, Wolfenden L, Francis JL, Sutherland R, et al. An implementation intervention to encourage healthy eating in centre-based child-care services: impact of the Good for Kids Good for Life programme. Public Health Nutr 2015 Jun;18(9):1610-1619. [doi: 10.1017/S1368980013003364] [Medline: 24477181]

20. Yoong SL, Skelton E, Jones J, Wolfenden L. Do childcare services provide foods in line with the 2013 Australian Dietary guidelines? A cross-sectional study. Aust N Z J Public Health 2014 Dec;38(6):595-596. [doi: 10.1111/1753-6405.12312] [Medline: 25440467]

21. Frampton AM, Sisson SB, Horm D, Campbell JE, Lora K, Ladner JL. What's for lunch? An analysis of lunch menus in 83 urban and rural Oklahoma child-care centers providing all-day care to preschool children. J Acad Nutr Diet 2014 Sep;114(9):1367-1374. [doi: 10.1016/j.jand.2013.09.025] [Medline: 24332085]

22. Wolfenden L, Finch M, Nathan N, Weaver N, Wiggers J, Yoong SL, et al. Factors associated with early childhood education and care service implementation of healthy eating and physical activity policies and practices in Australia: a cross-sectional study. Transl Behav Med 2015 Sep;5(3):327-334 [FREE Full text] [doi: 10.1007/s13142-015-0319-y] [Medline: 26327938]

23. Reeves P, Edmunds K, Szewczyk Z, Grady A, Yoong SL, Wolfenden L, et al. Economic evaluation of a web-based menu planning intervention to improve childcare service adherence with dietary guidelines. Implement Sci 2021 Jan 07;16(1):1 [FREE Full text] [doi: 10.1186/s13012-020-01068-x] [Medline: 33413491]

24. Vandelanotte C, Müller AM, Short CE, Hingle M, Nathan N, Williams SL, et al. Past, present, and future of eHealth and mHealth research to improve physical activity and dietary behaviors. J Nutr Educ Behav 2016 Mar;48(3):219-28.e1. [doi: 10.1016/j.jneb.2015.12.006] [Medline: 26965100]

25. Schoeppe S, Alley S, Van Lippevelde W, Bray NA, Williams SL, Duncan MJ, et al. Efficacy of interventions that use apps to improve diet, physical activity and sedentary behaviour: a systematic review. Int J Behav Nutr Phys Act 2016 Dec 07;13(1):127 [FREE Full text] [doi: 10.1186/s12966-016-0454-y] [Medline: 27927218]

26. Antwi F, Fazylova N, Garcon M, Lopez L, Rubiano R, Slyer J. The effectiveness of web-based programs on the reduction of childhood obesity in school-aged children: a systematic review. JBI Libr Syst Rev 2012;10(42 Suppl):1-14. [doi: 10.11124/jbisrir-2012-248] [Medline: 27820152]

27. Grady A, Wolfenden L, Wiggers J, Rissel C, Finch M, Flood V, et al. Effectiveness of a web-based menu-planning intervention to improve childcare service compliance with dietary guidelines: randomized controlled trial. J Med Internet Res 2020 Feb 04;22(2):e13401 [FREE Full text] [doi: 10.2196/13401] [Medline: 32014843]

28. Ward DS, Vaughn AE, Mazzucca S, Burney R. Translating a child care based intervention for online delivery: development and randomized pilot study of Go NAPSACC. BMC Public Health 2017 Nov 21;17(1):891 [FREE Full text] [doi: 10.1186/s12889-017-4898-z] [Medline: 29162057]

29. Bowen DJ, Kreuter M, Spring B, Cofta-Woerpel L, Linnan L, Weiner D, et al. How we design feasibility studies. Am J Prev Med 2009 May;36(5):452-457 [FREE Full text] [doi: 10.1016/j.amepre.2009.02.002] [Medline: 19362699]

30. Eldridge SM, Chan CL, Campbell MJ, Bond CM, Hopewell S, Thabane L, PAFS consensus group. CONSORT 2010 statement: extension to randomised pilot and feasibility trials. Pilot Feasibility Stud 2016 Oct 21;2:64 [FREE Full text] [doi: 10.1186/s40814-016-0105-8] [Medline: 27965879]

31. Curran G, Bauer M, Mittman B, Pyne J, Stetler C. Effectiveness-implementation hybrid designs: combining elements of clinical effectiveness and implementation research to enhance public health impact. Med Care 2012 Mar;50(3):217-226 [FREE Full text] [doi: 10.1097/MLR.0b013e3182408812] [Medline: 22310560]

32. Barnes C, Grady A, Nathan N, Wolfenden L, Pond N, McFayden T, et al. A pilot randomised controlled trial of a web-based implementation intervention to increase child intake of fruit and vegetables within childcare centres. Pilot Feasibility Stud 2020 Oct 29;6(1):163 [FREE Full text] [doi: 10.1186/s40814-020-00707-w] [Medline: 33292720]

33. HealthStats NSW. NSW Government. URL: https://www.healthstats.nsw.gov.au/\#/home [accessed 2021-11-28]

34. National registers. Australian Children's Education \& Care Quality Authority. URL: https://www.acecqa.gov.au/resources/ national-registers [accessed 2021-11-28]

35. Population Health Information Management System PHIMS, Healthy Children Initiative. Published 2019. Accessed 18 March 2019.

36. Finch M, Wolfenden L, Morgan PJ, Freund M, Wyse R, Wiggers J. A cluster randomised trial to evaluate a physical activity intervention among 3-5 year old children attending long day care services: study protocol. BMC Public Health 2010 Sep 08;10:534 [FREE Full text] [doi: 10.1186/1471-2458-10-534] [Medline: 20822543]

37. Socio-economic indexes for areas. Australian Bureau of Statistics. URL: https://www.abs.gov.au/websitedbs/censushome.nsf/ home/seifa [accessed 2021-11-28]

38. Michie S, van Stralen MM, West R. The behaviour change wheel: a new method for characterising and designing behaviour change interventions. Implement Sci 2011 Apr 23;6:42 [FREE Full text] [doi: 10.1186/1748-5908-6-42] [Medline: 21513547]

39. Powell BJ, Waltz TJ, Chinman MJ, Damschroder LJ, Smith JL, Matthieu MM, et al. A refined compilation of implementation strategies: results from the Expert Recommendations for Implementing Change (ERIC) project. Implement Sci 2015 Feb 12;10:21 [FREE Full text] [doi: 10.1186/s13012-015-0209-1] [Medline: 25889199] 
40. Wallace R, Costello L, Devine A. Exploring sense of community among early childhood education and care professionals through the Supporting Nutrition for Australian Childcare (SNAC) website. Australasian J Early Childhood 2018 Dec 01;43(4):23-32. [doi: 10.23965/ajec.43.4.03]

41. Proctor EK, Powell BJ, McMillen JC. Implementation strategies: recommendations for specifying and reporting. Implementation Sci 2013 Dec 1;8:139. [doi: 10.1186/1748-5908-8-139] [Medline: 24289295]

42. Seward K, Wolfenden L, Finch M, Wiggers J, Wyse R, Jones J, et al. Improving the implementation of nutrition guidelines in childcare centres improves child dietary intake: findings of a randomised trial of an implementation intervention. Public Health Nutr 2018 Feb;21(3):607-617. [doi: 10.1017/S1368980017003366] [Medline: 29173218]

43. Nathan N, Wiggers J, Bauman AE, Rissel C, Searles A, Reeves P, et al. A cluster randomised controlled trial of an intervention to increase the implementation of school physical activity policies and guidelines: study protocol for the physically active children in education (PACE) study. BMC Public Health 2019 Feb 11;19(1):170 [FREE Full text] [doi:

10.1186/s12889-019-6492-z] [Medline: 30760243]

44. Nathan N, Sutherland R, Hope K, McCarthy NJ, Pettett M, Elton B, et al. Implementation of a school physical activity policy improves student physical activity levels: outcomes of a cluster-randomized controlled trial. J Phys Act Health 2020 Sep 12;17(10):1009-1018. [doi: 10.1123/jpah.2019-0595] [Medline: 32919383]

45. Bélanger M, Humbert L, Vatanparast H, Ward S, Muhajarine N, Chow AF, et al. A multilevel intervention to increase physical activity and improve healthy eating and physical literacy among young children (ages 3-5) attending early childcare centres: the Healthy Start-Départ Santé cluster randomised controlled trial study protocol. BMC Public Health 2016 Apr 12;16:313 [FREE Full text] [doi: 10.1186/s12889-016-2973-5] [Medline: 27068684]

46. Adamo K, Wilson S, Harvey A, Grattan KP, Naylor PJ, Temple VA, et al. Does intervening in childcare settings impact fundamental movement skill development? Med Sci Sports Exerc 2016 May;48(5):926-932. [doi:

10.1249/MSS.0000000000000838] [Medline: 26656776]

47. Proctor E, Silmere H, Raghavan R, Hovmand P, Aarons G, Bunger A, et al. Outcomes for implementation research: conceptual distinctions, measurement challenges, and research agenda. Adm Policy Ment Health 2011 Mar;38(2):65-76 [FREE Full text] [doi: 10.1007/s10488-010-0319-7] [Medline: 20957426]

48. Welcome to Google Analytics. Analytics. URL: https://analytics.google.com/analytics/web/provision/\#/provision [accessed 2021-11-28]

49. Yoong S, Grady A, Wiggers J, Stacey FG, Rissel C, Flood V, et al. Child-level evaluation of a web-based intervention to improve dietary guideline implementation in childcare centers: a cluster-randomized controlled trial. Am J Clin Nutr 2020 Apr 01;111(4):854-863 [FREE Full text] [doi: 10.1093/ajcn/nqaa025] [Medline: 32091593]

50. Weiner BJ, Lewis CC, Stanick C, Powell BJ, Dorsey CN, Clary AS, et al. Psychometric assessment of three newly developed implementation outcome measures. Implement Sci 2017 Aug 29;12(1):108 [FREE Full text] [doi: 10.1186/s13012-017-0635-3] [Medline: 28851459]

51. Jones J, Wyse R, Finch M, Lecathelinais C, Wiggers J, Marshall J, et al. Effectiveness of an intervention to facilitate the implementation of healthy eating and physical activity policies and practices in childcare services: a randomised controlled trial. Implement Sci 2015 Oct 25;10:147 [FREE Full text] [doi: 10.1186/s13012-015-0340-z] [Medline: 26498746]

52. Children's Services Award 2010. Fair Work OMBUDSMAN. URL: https://awardviewer.fwo.gov.au/award/show/MA000120 [accessed 2021-11-28]

53. Ward DS, Mazzucca S, McWilliams C, Hales D. Use of the Environment and Policy Evaluation and Observation as a Self-Report Instrument (EPAO-SR) to measure nutrition and physical activity environments in child care settings: validity and reliability evidence. Int J Behav Nutr Phys Act 2015 Sep 26;12:124 [FREE Full text] [doi: 10.1186/s12966-015-0287-0] [Medline: 26410387]

54. Dodds P, Wyse R, Jones J, Wolfenden L, Lecathelinais C, Williams A, et al. Validity of a measure to assess healthy eating and physical activity policies and practices in Australian childcare services. BMC Public Health 2014 Jun 09;14:572 [FREE Full text] [doi: 10.1186/1471-2458-14-572] [Medline: 24909075]

55. Damschroder LJ, Aron DC, Keith RE, Kirsh SR, Alexander JA, Lowery JC. Fostering implementation of health services research findings into practice: a consolidated framework for advancing implementation science. Implement Sci 2009 Aug 07;4:50 [FREE Full text] [doi: 10.1186/1748-5908-4-50] [Medline: 19664226]

56. StataCorp. STATA. 4905 Lakeway Drive, College Station, TX 77845 USA 2020:2020.

57. The Australian Statistical Geography Standard (ASGS) Remoteness Structure. Australian Bureau of Statistics. 2012. URL: https://www.nintione.com.au/resources/rao/remoteness-structure/ [accessed 2021-11-28]

58. Dev DA, Byrd-Williams C, Ramsay S, McBride B, Srivastava D, Murriel A, et al. Engaging parents to promote children's nutrition and health. Am J Health Promot 2017 Mar;31(2):153-162. [doi: 10.1177/0890117116685426] [Medline: 28423928]

59. van de Kolk I, Goossens A, Gerards S, Kremers S, Manders R, Gubbels J. Healthy nutrition and physical activity in childcare: views from childcare managers, childcare workers and parents on influential factors. Int J Environ Res Public Health 2018 Dec 19;15(12):2909 [FREE Full text] [doi: 10.3390/ijerph15122909] [Medline: 30572600]

60. Finch M, Wolfenden L, Morgan PJ, Freund M, Jones J, Wiggers J. A cluster randomized trial of a multi-level intervention, delivered by service staff, to increase physical activity of children attending center-based childcare. Prev Med 2014 Jan;58:9-16. [doi: 10.1016/j.ypmed.2013.10.004] [Medline: 24145204] 
61. Hardy LL, King L, Kelly B, Farrell L, Howlett S. Munch and Move: evaluation of a preschool healthy eating and movement skill program. Int J Behav Nutr Phys Act 2010 Nov 03;7:80 [FREE Full text] [doi: 10.1186/1479-5868-7-80] [Medline: 21047434]

62. Langford R, Bonell C, Jones H, Pouliou T, Murphy SM, Waters E, et al. The WHO Health Promoting School framework for improving the health and well-being of students and their academic achievement. Cochrane Database Syst Rev 2014 Apr 16(4):CD008958. [doi: 10.1002/14651858.CD008958.pub2] [Medline: 24737131]

63. National quality standard. Australian Children's Education \& Care Quality Authority. URL: https://www.acecqa.gov.au/ nqf/national-quality-standard [accessed 2021-11-28]

64. Finch M, Wolfenden L, Falkiner M, Edenden D, Pond N, Hardy LL, et al. Impact of a population based intervention to increase the adoption of multiple physical activity practices in centre based childcare services: a quasi experimental, effectiveness study. Int J Behav Nutr Phys Act 2012 Aug 29;9:101 [FREE Full text] [doi: 10.1186/1479-5868-9-101] [Medline: 22929434]

65. Luecking CT, Dobson P, Ward DS. Barriers and facilitators of parent engagement with health promotion in child care: a mixed-methods evaluation. Health Educ Behav 2020 Dec;47(6):914-926. [doi: 10.1177/1090198120952040] [Medline: $\underline{32815417]}$

\author{
Abbreviations \\ BCT: behavior change technique \\ BCW: Behavior Change Wheel \\ EATS: Electronic Assessment Tool and Support \\ ECEC: early childhood education and care \\ Go-NAPSACC: Go-Nutrition and Physical Activity Self-Assessment for Child Care \\ HNE: Hunter New England \\ HPO: health promotion officer \\ MoU: memorandum of understanding \\ NSW: New South Wales \\ RCT: randomized controlled trial \\ SES: socioeconomic status
}

Edited by G Eysenbach; submitted 03.12.20; peer-reviewed by C Saldaña, H Belli; comments to author 14.02.21; revised version
received 08.03.21; accepted 05.11.21; published 15.12.21
Please cite as:
Barnes C, Yoong SL, Nathan N, Wolfenden L, Wedesweiler T, Kerr J, Ward DS, Grady A
Feasibility of a Web-Based Implementation Intervention to Improve Child Dietary Intake in Early Childhood Education and Care:
Pilot Randomized Controlled Trial
J Med Internet Res $2021 ; 23(12): e 25902$
URL: $\underline{\text { https://www.jmir.org/2021/12/e25902 }}$
doi: $10.2196 / 25902$
PMID:

(C) Courtney Barnes, Sze Lin Yoong, Nicole Nathan, Luke Wolfenden, Taya Wedesweiler, Jayde Kerr, Dianne S Ward, Alice Grady. Originally published in the Journal of Medical Internet Research (https://www.jmir.org), 15.12.2021. This is an open-access article distributed under the terms of the Creative Commons Attribution License (https://creativecommons.org/licenses/by/4.0/), which permits unrestricted use, distribution, and reproduction in any medium, provided the original work, first published in the Journal of Medical Internet Research, is properly cited. The complete bibliographic information, a link to the original publication on https://www.jmir.org/, as well as this copyright and license information must be included. 